\title{
Learning and Upgrading in Global Value Chains An Analysis of India's Manufacturing Sector
}

\section{Sourish Dutta}

\author{
CDS, Trivandrum
}




\section{Outline}

- Part I: Stylised facts of Global Value Chain (GVC)

- Concept - Structure - Formation - Impacts

- Part II: Modeling Learning \& Upgrading in GVCs

- Motivation

- Fundamentals

- Conceptualisation

- Structuralisation

- Formation

- Impacts 


\section{Part I}

Stylised facts of Global Value Chain (GVC)

Concept - Structure - Formation - Impacts 


\section{Concept (WDR 2020)}

- What is a Global Value Chain (GVC)?

- Series of stages in the production of a product... Chain

- Firms specialise in a specific stage adding value... Value

- At least two stages are in different countries... Global

- A product assembled in $C_{1}$ with parts from $C_{2} \& C_{3}$, and exported to $C_{4}$ is a GVC

- By this definition, a country, sector, or firm participates in a GVC if it engages in (at least) one stage in a GVC.

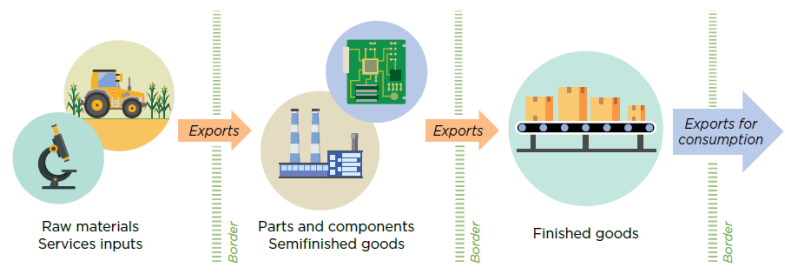




\section{Where do bicycles come from? (WDR 2020)}

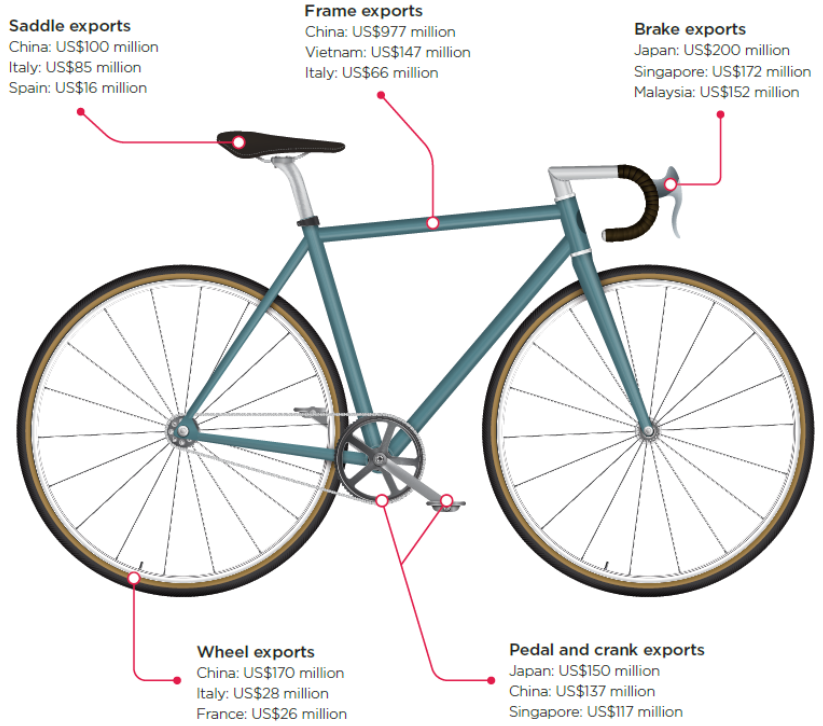




\section{Structure (WDR 2020)}

- It is often associated with either international trade...

- in raw materials (such as tin or aluminum),

- in intermediate inputs (such as car parts),

- or in tasks (such as back-office services).

- Various configurations that a GVC might take...

- Spider-like structures - multiple parts converging at a point

- Snake-like structures - value created sequentially in a series of stages

- Fragmenting production across borders gives rise to...

- A finer international division of labour

- Greater productive use of resources across countries, sectors, and stages of production 


\section{Formation (WDR 2020)}

- How do GVCs work?

- Interactions between firms typically involve durable relationships

- Economic fundamentals drive countries' participation in GVCs

- But policies matter-to enhance participation and broaden benefits

- This complex approach to trade leads to rich set of determinants...

- Endowments, Market size, Geography, Institutions

- Challenges for measuring...

- Macro view - tracing value-added trade flows across countries and sectors - global input-output tables with homogeneity and proportionality assumptions

- Micro view - Product-level - firm-level data capturing the heterogeneity in GVC linkages across firms - organising production networks 


\section{Impacts (WDR 2020)}

- GVC participation leads to a detailed set of consequences...

- GVC trade crossing borders multiple times magnifying the growth, employment, and distributional impacts of standard trade

- Challenging consequences...

- Gains from GVC participation may be distributed unequally within and across countries

- In some countries and sectors, firms could be locked in in relatively low value-added segments of production with little scope for upgrading.

- GVCs (relational) can facilitate industrialisation by...

- Reducing the range of "capabilities" required to produce and export

- Learning - acquiring new capabilities - know-how/technologies/skills

- Upgrading (innovation) - transition from simple production tasks to specialising in intangible capital and breaking into new industries 


\section{Part II}

\section{Modeling Learning \& Upgrading in GVCs}

Motivation - Fundamentals - Concept - Structure - Formation - Impacts 


\section{Motivation (1): Dynamic Comparative Advantage}

- In 2015, Stiglitz (with Arrow, Solow, and Aghion) redefined the theory of comparative advantage...

- From static to dynamic using Arrow's "Learning" perspective

- Creating a Learning Society: A New Approach to Growth, Development, and Social Progress (Columbia University Press)

- Mechanics of Global Value Chains - Learning and Upgrading

It has become conventional wisdom to emphasize that what matters is not static comparative advantage but dynamic comparative advantage. Korea did not have a comparative advantage in producing semiconductors when it embarked on its transition. Its static comparative advantage was in the production of rice. Had it followed its static comparative advantage (as many neoclassical economists had recommended) then that might still be its comparative advantage; it might be the best rice grower in the world, but it would still be poor. But a country's dynamic comparative advantage is endogenous, a result of what it does. There seems to be a circularity here. The central question is, What should a country do today to create its dynamic comparative advantage? 


\section{Motivation (2): Arrow's Comments}

- Comment on Persistence of Inefficiencies... Differences in Productivity

- "I will concentrate on the issue of learning and knowledge... since this is a topic of great interest to me... issue of knowledge spillovers and their effects on growth is not necessarily limited to a discussion of foreign trade at all."

- "What we know about spillovers is that they tend to be local and that therefore geography matters... information and learning happen most effectively when people meet face-to-face and exchange ideas. The interesting thing about knowledge is that it has an ability to spread rapidly and effectively... individuals and firms are lighting candles all over the place."

- "One of the advantages of trade is that it facilitates the spreading of information of technological knowledge. The fact that there is contact - that ideas are flowing - leads to spillover effects, learning, and greater competition." 


\section{Fundamentals (1): Connecting the Dots}

- Modeling GVC links

- O-ring theory... Sequential production

- Learning theory... Acquisition of knowledge

- Network theory... Interaction (collective)

- Sequential production process... Multiple equilibria

- Production requires the completion of a series of tasks

- Mistakes in any of which can dramatically reduce the product's value

- Learning and upgrading... Non-equilibrium

- Efficiency gains from knowledge spillovers

- "Knowledge is a free good. The biggest cost in its transmission is not the production or distribution of knowledge, but in its assimilation. This is something that all teachers know."

- "Kenneth Arrow... showing point by point how general equilibrium did not match with real economies... he was willing to reassess his own ideas if that furthered the truth" 


\section{Fundamentals (2): The Value in the Links}

- Products are vehicles for knowledge

- Markets and organisations allow the knowledge to spread

- The amount of knowledge embedded in a production process depends on...

- the diversity of knowledge across individuals... distribution

- their ability to combine this knowledge and make use of it... interactions 


\section{Framing "The Value in the Links"}

- The Conceptualisation of GVC Links... Definitions

- The Structure of GVC Links... Trends \& Patterns

- The Formation of GVC Links... Drivers

- Shaping Economic \& Social Outcomes... Impacts 


\section{Conceptualisation (1)}

- Economies tend to diversify, rather than specialise, as the number of available capabilities grows

- Economies that are able to utilise rare capabilities or combine many capabilities to make complex products tend to be richer and grow more quickly

- Economies tend to find new products to produce that leverage their existing capabilities. 


\section{Conceptualisation (2)}

- Three properties of knowledge and learning individually:

- Limited capacity to reason i.e. bounded rationality

- Limited capacity to learn

- Limited capacity to transmit experiential knowledge

- Limited learnability... why sometimes knowledge does not move freely and concentrate in few pockets

- It is hard for individuals to learn... collectives (firms, industries, countries) can learn by gathering people

- Economies that are participating in various production networks accumulate adequate capabilities... productivity 


\section{Structuralisation (1)}

- Let Indian economic activities be indexed by $p$ (i.e. industry specific output or product $p$ )

- Production units (i.e. firms) $\rightarrow f$

- $X_{f, p}$ is a variable that takes value 1 if the firm $f$ is capable to produce $p$ successfully in India, and 0 if not.

- Here our objective is to find out the expression of probability that a production unit or firm $f$ is capable to produce product in India: $\operatorname{Pr}\left(X_{f, p}=1\right)$

- $C_{p}(\gg 1) \rightarrow$ stages of processing associated with the production of $p$.

- Let $c_{f}$ be the probability that the firm $f$ has any random capability of the $C_{p}$ capabilities required for the $p$ production.

- Suppose India provides $C$ capabilities to the firm $f$ (i.e. $0 \leq C \leq C_{p}$ ) \& probability that India provides any of the capabilities is $C$ 


\section{Structuralisation (2)}

$\operatorname{Pr}\left(\right.$ firm $f$ in India produces $p \mid$ India facilitates $C$ of the $C_{p}$ capabilities)

$$
\begin{aligned}
\operatorname{Pr} & \left(X_{f, p}=1 \mid C\right)=c_{f} C_{p}-C \\
\operatorname{Pr}\left(X_{f, p}=1\right)= & \sum_{C=0}^{C_{p}} \operatorname{Pr}\left(X_{f, p}=1 \mid C\right) \operatorname{Pr}(C) \\
= & \sum_{C=0}^{C_{p}} c_{f} C_{p}-C\left(\begin{array}{c}
C_{p} \\
C
\end{array}\right){ }^{C}(1-c)^{C_{p}-C} \\
= & \sum_{C=0}^{C_{p}}{ }^{C}{ }^{C}\left(c_{f}(1-c)\right)^{C_{p}-C} \\
= & \left(c+c_{f}(1-c)\right)^{C_{p}} \\
= & \left(1-\left(1-c_{f}\right)(1-c)\right)^{C_{p}}
\end{aligned}
$$




\section{Structuralisation (3)}

The complementarity between the firm, the economy and the activity

$$
\phi\left(C_{p}, C_{f}, c\right) \approx e^{-C_{p}\left(1-c_{f}\right)(1-c)}
$$

- $C_{p}$ : This is the number of capabilities required to produce the industry-specific product $\mathrm{p}$. We can refer to it as the complexity of the product.

- $1-c_{f}$ : This is the firm-specific probability of lacking any of the capabilities required in production processes. Conversely, $c_{f}$ can be referred to as a measure of individual knowledge.

- $1-c$ : This is the India-specific probability of lacking any of the capabilities required in production processes. Conversely, $c$ can be referred to as a measure of collective knowledge and represents a measure of input availability, which in turn represents a measure of India's diversity. 


\section{Structuralisation (4)}

- A large set of inputs must be combined to generate an output. The output will not be produced, however, if any of the inputs is missing.

- This is a specific form of a production function called the Leontief production function - presence of an output follows a logic of complementarity.

- May or may not be produced (stochastic) - Scope of quality

- Mathematically, complementarity meant taking products of probabilities. Thus, it can also be said that outputs follow a logic of multiplicativity

- We can claim that the logic of multiplicativity between inputs for determining the presence of an output, in turn, implies that the presence of an input follows a logic of additivity between outputs. 


\section{Structuralisation (5)}

- $\operatorname{Pr}\left(\right.$ firm $f$ in India produces $p \mid$ India facilitates $C$ of the $C_{p}$ capabilities) is the product $c_{f} c_{f} \ldots c_{f}$

- It is the probability of having the first capability times the probability of having the second, and so on, until we have the probability of getting each of the missing capabilities not offered in India.

- Central limit theorem - When the outcome is produced by many small effects acting additively and independently, its distribution will be close to normal.

- Not valid if the effects act multiplicatively or if there is a single external influence that has a considerably larger magnitude than the rest of the effects.

- Our counting problem includes a discrete to continuum approx, infinitely divisible and decomposable distributions

- The central limit theorem: Binomial $\rightarrow$ Normal $\rightarrow$ Log-normal (exponential of normal) 


\section{Structuralisation (6)}

- Set of Capabilities/Skills $-C_{p}$ for $p$

- Public $\mathrm{g} / \mathrm{s}(C)$ and Private $\mathrm{g} / \mathrm{s}\left(C_{p}-C\right)$

- Public probability $c$ and Private probability $c_{f}$

- GVC trade $\rightarrow$ Trade in tasks/parts

- Product... Interaction of firms

$$
\begin{gathered}
\operatorname{Pr}\left(X_{f, p}=1\right)=\phi\left(C_{p}, c_{f}, c\right) \approx e^{-C_{p}\left(1-c_{f}\right)(1-c)} \\
\sum_{i} \phi=\gamma \rightarrow G \beta
\end{gathered}
$$

- In Bayesian inference, the gamma distribution is the conjugate prior to... 


\section{Formation (1)}

- Non-equilibrium theory for the sequential Production Process (SPP)

- Production $=$ Driving (Learning) + Relaxing (Upgrading)

- As process unfolds less and less stages can be reached

- Often backward stages constrain possibilities for forward stages

- Production processes become more constrained as they unfold

$$
\Omega_{1} \subset \Omega_{2} \subset \Omega_{3} \ldots \subset \Omega_{n}
$$

- Restart means $\Omega_{1}=\Omega_{n}$

- Sequential Production Process (SPP) 


\section{Formation (2): Zipf 's law emerges}

- This is seen by the following simple argument. The probability of arriving at stage $i$ is computed by $p_{i}=\sum_{j}^{n} p(i \mid j) p_{j}$

- Therefore, the transition probability from stage $j$ to $i$ is

$$
p(i \mid j)=\frac{1}{j-1}, i<j
$$

- Inserting $p(i \mid j)$ to $p_{i}=\sum_{j}^{n} p(i \mid j) p_{j}$, we get for the difference $p_{i+1}-p_{i}$,

$$
p_{i+1}-p_{i}=-\frac{p_{i+1}}{i}
$$

- If we read this equation in differential form, $\frac{d p_{i}}{d i}=-\frac{p_{i}}{i}$, we see immediately that $\log p_{i}=-\log i$, and we get the result, $p_{i}=i^{-1}$

- SPP lead to exact Zipf's law! 


\section{Formation (3): Zipf 's law emerges}

- Up to now we have assumed that every stage that is after the present stage $i$ is visited with the same probability $\frac{1}{i-1}$. This is not necessary and we can assume a priori probabilities $q_{i}$ for every stage $i$. In this case, we get for the transition probability

$$
p(i \mid j)=\frac{q_{i}}{g_{i}-1}, i<j
$$

where $g_{i}=\sum_{j=1}^{j} q_{j}$. Remarkably, for a wide range of choices of $q_{i}$, the same result for the visiting probabilities is obtained, $p_{i}=i^{-1}$. 


\section{Formation (4): The influence of the driving rate}

- We can now ask what happens if we alter the driving rate of the process. In particular, if we allow the process to be restarted during its relaxation towards equilibrium.

- In the example shown before, we would bring the process to its starting level at any time before it reaches the last stage. Assume that we restart the process at any state with a probability of $1-\lambda$, then we get for the transition rate,

$$
\begin{aligned}
p(i \mid j)= & \frac{\lambda}{j-1}+\frac{1-\lambda}{n}, i<j \\
= & \frac{1-\lambda}{n}, i \geq j>1 \\
& =\frac{1}{n}, i \geq j=1
\end{aligned}
$$

Using the same procedure as before, we get the recursive relation which yields an exact power law, $p_{i}=i^{-\lambda}$. 


\section{Formation (5)}

- Zipf's law emerges

- Distributions of SPP processes converge to Zipf's law as a limit distribution $p_{i}=i^{-1}$, regardless of the priori probabilities of visiting the individual states. Zipf's law acts as an attractor.

- The influence of the driving rate $p_{i}=i^{-\lambda}$

- This means that SPP processes lead to exact power law distributions in the visiting times of states, with an exponent that is one minus the driving (or reset) rate, $\lambda$. This happens if the process is driven before it reaches its equilibrium state or, in other words, if it does not have the time to fully relax before it is excited again.

- Stage-dependent driving rates and the emergence of statistics

$$
\lambda(x)=-x \frac{d}{d x} \log p(x)
$$

- A stage-dependent driving rate $1-\lambda(i)$ allows us to obtain practically every distribution function that occurs in this context. 


\section{Shaping Economic \& Social Outcomes (1)}

Technological improvement of production process of $p$ :

$$
\frac{\frac{\partial \phi\left(C_{p}, c_{f}, c\right)}{\partial\left(-C_{p}\right)}}{\phi\left(C_{p}, c_{f}, c\right)}=\left(1-c_{f}\right)(1-c)
$$

Individual learning for firm $f$ :

$$
\frac{\frac{\partial \phi\left(C_{p}, c_{f}, c\right)}{\partial\left(C_{p} c_{f}\right)}}{\phi\left(C_{p}, c_{f}, c\right)}=(1-c)
$$

Collective learning for India:

$$
\frac{\frac{\partial \phi\left(C_{p}, c_{f}, c\right)}{\partial\left(C_{p} c\right)}}{\phi\left(C_{p}, c_{f}, c\right)}=\left(1-c_{f}\right)
$$




\section{Shaping Economic \& Social Outcomes (2)}

- India is a collective, the probability it provides an input is larger than the probability an individual has it, so $c \gg c_{f}$. Conversely, $1-c \ll 1-c_{f}$.

- Consequently, we have that $0<(1-c)\left(1-c_{f}\right)<(1-c) \ll\left(1-c_{f}\right)$

- The implication is that these rates have the following order:

$$
0<\frac{\frac{\partial \phi\left(C_{p}, c_{f}, c\right)}{\partial\left(-C_{p}\right)}}{\phi\left(C_{p}, C_{f}, c\right)}<\frac{\frac{\partial \phi\left(C_{p}, c_{f}, c\right)}{\partial\left(C_{p} c_{f}\right)}}{\phi\left(C_{p}, C_{f}, c\right)} \ll \frac{\frac{\partial \phi\left(C_{p}, c_{f}, c\right)}{\partial\left(C_{p} c\right)}}{\phi\left(C_{p}, C_{f}, c\right)}
$$

- Effect of technological improvement $<$ Effect of individual learning $\ll$ Effect of collective learning.

- Intuitively, thus, it is easy to see that increases in collective knowledge have a reinforcing effect and suggest a virtuous cycle 


\section{Shaping Economic \& Social Outcomes (3)}

- Taking negative logarithms twice in (3) yields

$$
-\ln \left(-\ln \left(\phi\left(C_{p}, C_{f}, c\right)\right)\right)=-\ln \left(C_{p}\right)-\ln \left(1-c_{f}\right)-\ln (1-c)
$$

- If one has the means to estimate the probability on the left-hand side, $\left.\widehat{(} \phi\left(C_{p}, C_{f}, c\right)\right)$, then one can regress this "double-logged-likelihood" against three additive fixed-level effects from the production, the firm, and the economy,

$$
\left.-\ln \left(-\ln \left(\widehat{(\phi}\left(C_{p}, C_{f}, c\right)\right)\right)\right)=\alpha_{p}+\beta_{f}+\gamma+\epsilon_{p, f}
$$

- where $\epsilon_{p, f}$ is the error one would try to minimise in the estimation of the fixed effects. 